\title{
Modélisation, identification et simulation d'un séchoir solaire à bois
}

\author{
D. Pallet $(*)$, M. Fournier $\left({ }^{*}\right)$ et A. Themelin $\left({ }^{* *}\right)$ \\ (*) Institut de Sciences et Génie des Matériaux et Procédés, UP32, CNRS, Université de Perpignan, avenue \\ de Villeneuve, 66025 Perpignan, France \\ ${ }^{(* *)}$ Centre d'Etudes et d'Expérimentation du Machinisme Agricole Tropical, CIRAD, Montpellier, France
}

(Reçu le 2 février 1987, révisé le 19 juin 1987, accepté le 20 juillet 1987)

\begin{abstract}
Résumé. - Un modèle mathématique décrivant le comportement d'un séchoir solaire à bois construit à Kourou (Guyane) est présenté. Conçu en deux parties, ce modèle représente tout d'abord les phénomènes de migration et d'évaporation de l'eau dans le bois. Les paramètres des équations sont identifiés à partir de mesures effectuées en laboratoire. La modélisation globale du séchoir incluant le modèle précédent permet ensuite une définition de régulation adaptée au cycle solaire. Une opération de séchage d'une essence de bois particulière (l'Angélique) est simulée dans des conditions climatiques données. Le programme qui résulte de cette étude permet une optimisation de la structure et du fonctionnement du séchoir actuel. Il constitue aussi un outil d'aide à la conception de nouveaux séchoirs.
\end{abstract}

\begin{abstract}
A mathematical model for describing the behaviour of a solar timber dryer built at Kourou (French Guyana) is being presented. This model consists of two parts : - in the first the movement and evaporation of water in wood are studied. The parameters used in the equation are derived from experimental laboratory data - in the second, the total dryer model including the part presented above, can provide information pertaining to the regulation of an adapted solar cycle. By employing this model, the drying of Angelic wood has been simulated under tropical climates. Our results can be employed to optimise the design and handling of a specific solar dryer. This model can be used as a tool in the conception of new dryers.
\end{abstract}

\section{Nomenclature}

$\begin{array}{lll}a, b, c, d, e, f, g: \text { paramètres du modèle évaporatoire } & (-) \\ C & : \text { concentration } & \left(\mathrm{kg} \cdot \mathrm{m}^{-3}\right) \\ C_{\mathrm{p}} & : \text { chaleur massique à pression constante } & \left(\mathrm{J} \cdot \mathrm{kg}^{-1} \cdot{ }^{\circ} \mathrm{C}^{-1}\right) \\ D_{\mathrm{m}} & : \text { coefficient de diffusion massique } & \left(\mathrm{m}^{2} \cdot \mathrm{s}^{-1}\right) \\ D_{\mathrm{h}} & : \text { diamètre hydraulique ou longueur caractéristique } \\ E & : \text { énergie d'activation } & (\mathrm{m}) \\ e p & : \text { épaisseur parois cellule } & (\mathrm{J}) \\ E_{\mathrm{b}} & : \text { épaisseur des planches } & (\mathrm{m}) \\ E_{\mathrm{fe}} & : \text { épaisseur du film d'eau condensée } & (\mathrm{m}) \\ F & : \text { fonctions } & (\mathrm{m}) \\ g & : \text { accélération terrestre } & \left(\mathrm{m} . \mathrm{s}^{-2}\right) \\ H & : \text { enthalpie } & (\mathrm{J}) \\ H_{\mathrm{a}} & : \text { enthalpie air interne à la cellule } & (\mathrm{J}) \\ H_{\mathrm{ee}} & : \text { enthalpie air venant de l'extérieur vers la cellule pendant } \Delta t & (\mathrm{~J}) \\ H_{\mathrm{ec}} & : \text { enthalpie air venant des capteurs vers la cellule pendant } \Delta t & (\mathrm{~J}) \\ H_{\mathrm{se}} & : \text { enthalpie air sortant de la cellule vers l'extérieur pendant } \Delta t & (\mathrm{~J}) \\ H_{\mathrm{sc}} & : \text { enthalpie air sortant de la cellule vers les capteurs pendant } \Delta t & (\mathrm{~J}) \\ H H & : \text { hauteur parois cellule } & (\mathrm{m}) \\ K_{1} & : \text { coefficient } & (-)\end{array}$




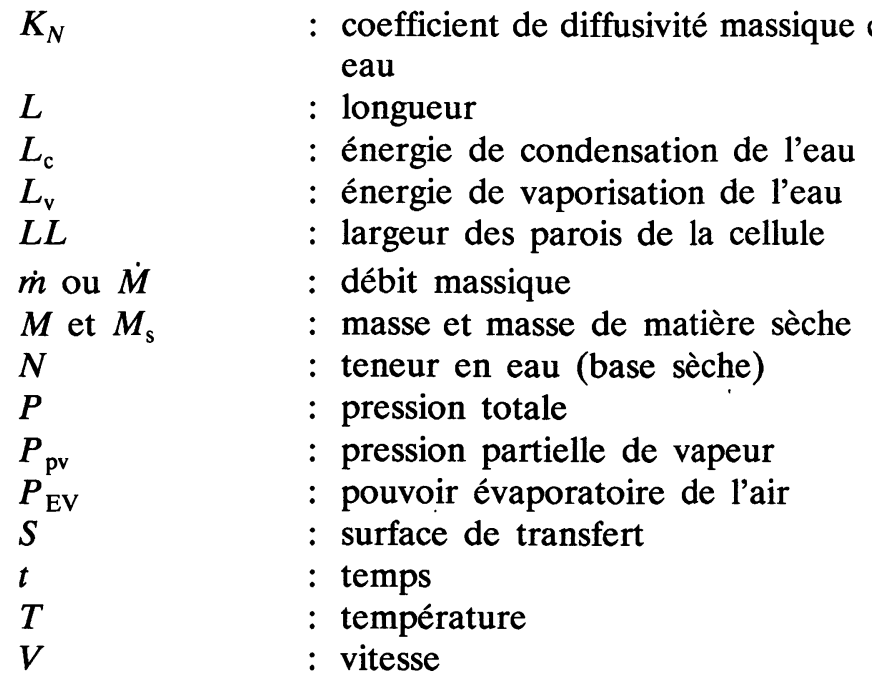

$\left(\mathrm{kg} \cdot \mathrm{m}^{-1} \cdot \mathrm{s}^{-1}\right)$

(m)

(J. $\left.\mathrm{kg}^{-1}\right)$

(J. $\left.\mathrm{kg}^{-1}\right)$

(m)

(kg. $\left.\mathrm{s}^{-1}\right)$

(kg)

$(-)$

(Pa)

(Pa)

(m. $\left.\mathrm{s}^{-1}\right)$

\section{Lettres Grecques}

$\alpha$

$\beta$

$\Delta t$

$\Delta t$

$\lambda$

$\delta$

$\nu$

$\rho$

\section{Indices}

b

c

cap

cel

cr

e

e

eq

ex

f

i

init.

m

o

$\mathrm{p}$

per

r

s

sat

surf

th

tab

v
: coefficient de transfert de chaleur

: coefficient de transfert de masse

: intervalle de temps

: intervalle de température

: conductivité thermique

coefficient de thermomigration

viscosité cinématique de l'air

: masse volumique
(W. $\mathrm{m}^{-2}{ }^{\circ} \mathrm{C}^{-1}$ ) (m. $\mathrm{s}^{-1}$ )

(s)

$\left({ }^{\circ} \mathrm{C}\right)$

W. $\left.\mathrm{m}^{-1} \cdot{ }^{\circ} \mathrm{C}^{-1}\right)$

$\left(\mathrm{m}^{2} \cdot \mathrm{s}^{-1}\right)$

$\left(\mathrm{m}^{2} \cdot \mathrm{s}^{-1}\right)$

(kg. $\mathrm{m}^{-3}$ )

\section{Nombres adimensionnels}

$\begin{array}{lll}\mathrm{Nu} & : \text { Nusselt } & \left(\mathrm{Nu}=\alpha \cdot D_{\mathrm{h}} / \lambda\right) \\ \mathrm{Pr} & : \text { Prandlt } & \left(\mathrm{Pr}=\nu / D_{\mathrm{m}}\right) \\ \mathrm{Re} & : \text { Reynolds } & \left(\operatorname{Re}=V \cdot D_{\mathrm{h}} / \nu\right)\end{array}$

\section{Introduction.}

L'étude du fonctionnement du séchoir solaire met en évidence la nécessité d'une étude dynamique du système. En effet plusieurs phénomènes cycliques sont présents simultanément.

- Au niveau d'un jour, le cycle solaire impose sa propre fréquence mais les périodes d'arrêt de la ventilation des capteurs commandés par la régulation doivent être prises en compte dans la gestion énergétique du système.

- Au niveau du cycle de séchage, l'apport énergétique est moyenné sur plusieurs jours, alors que la vitesse de séchage évolue dans de fortes proportions en quelques heures. La durée réelle de ce cycle reste à définir.

Ces remarques soulèvent de nombreuses questions relatives au fonctionnement énergétique du système. Deux approches sont possibles pour obtenir ces informations.

- La construction d'un séchoir et son expérimentation : ceci a été réalisé à Kourou (Guyane).

- La modélisation mathématique qui permet de simuler le fonctionnement du séchoir en régime dynamique et d'extrapoler à des conditions particulières.

C'est la deuxième voie que nous présentons ici : la comparaison des résultats théoriques et expérimen- 
taux, devant permettre de préciser.le modèle et de le recaler sur la réalité.

Notre étude étant davantage basée sur la connaissance du séchoir que sur celle du produit séché, une méthode déductive a été utilisée : elle consiste à décomposer la vitesse de séchage en fonctions invariantes.

Ce procédé permet de bien visualiser l'influence de chacun des paramètres intervenant dans une opération de séchage.

\section{Présentation du séchoir étudié.}

Le séchoir sur les caractéristiques duquel nous allons élaborer notre modèle est construit à Kourou (Guyane). Le cahier des charges servant de base à cette conception stipule que 8 à $10 \mathrm{~m}^{3}$ de sciages verts doivent être séchés jusqu'à $12 \%$ de teneur en eau en 20, 30 et 45 jours, respectivement pour des épaisseurs de 30,40 et $45 \mathrm{~mm}$ brut de sciage (Themelin [1]).

La structure de base est classique pour ce type de séchoir. Une enceinte close et isolée à renouvellement d'air, utilisant un échangeur de chaleur constitue la cellule ; des capteurs solaires fonctionnant en boucle fermée sur celle-ci, jouent le rôle du générateur de chaleur (Fig. 1)

Le volume intérieur de la cellule est de $65 \mathrm{~m}^{3}$; l'empilement des débits de sciage sur baguettes permet le passage de l'air, la pile peut occuper des dimensions maximales de : $L=4,6 \mathrm{~m} ; l=1,5 \mathrm{~m}$; $h=2,3 \mathrm{~m}$. Autour des planches sont agencés les principaux éléments aérauliques : couloirs de circulation d'air, faux plafonds, ventilateurs de brassage. Des cloisons articulées jouxtant la pile séparent l'écoulement d'air en trois parties pour obtenir un séchage du bois plus homogène. Chaque tiers de séchoir possède un ventilateur de brassage, un système de renouvellement d'air, et une partie des capteurs solaires. L'ensemble est réalisé avec des éléments industriels : ossature bois, panneaux métalliques isolés par injection de polyuréthane $(12 \mathrm{~cm})$. Un échangeur de chaleur doit être placé ultérieurement sur le circuit de renouvellement d'air, et récupérer ainsi une partie de l'énergie contenue dans l'air rejeté.

La surface de captage, disposée au-dessus de la cellule est inclinée de $10^{\circ}$ par rapport à l'horizontale et orientée vers le Sud (Kourou est à $5^{\circ}$ de latitude Nord). L'ensemble d'une surface de $75 \mathrm{~m}^{2}$ repose

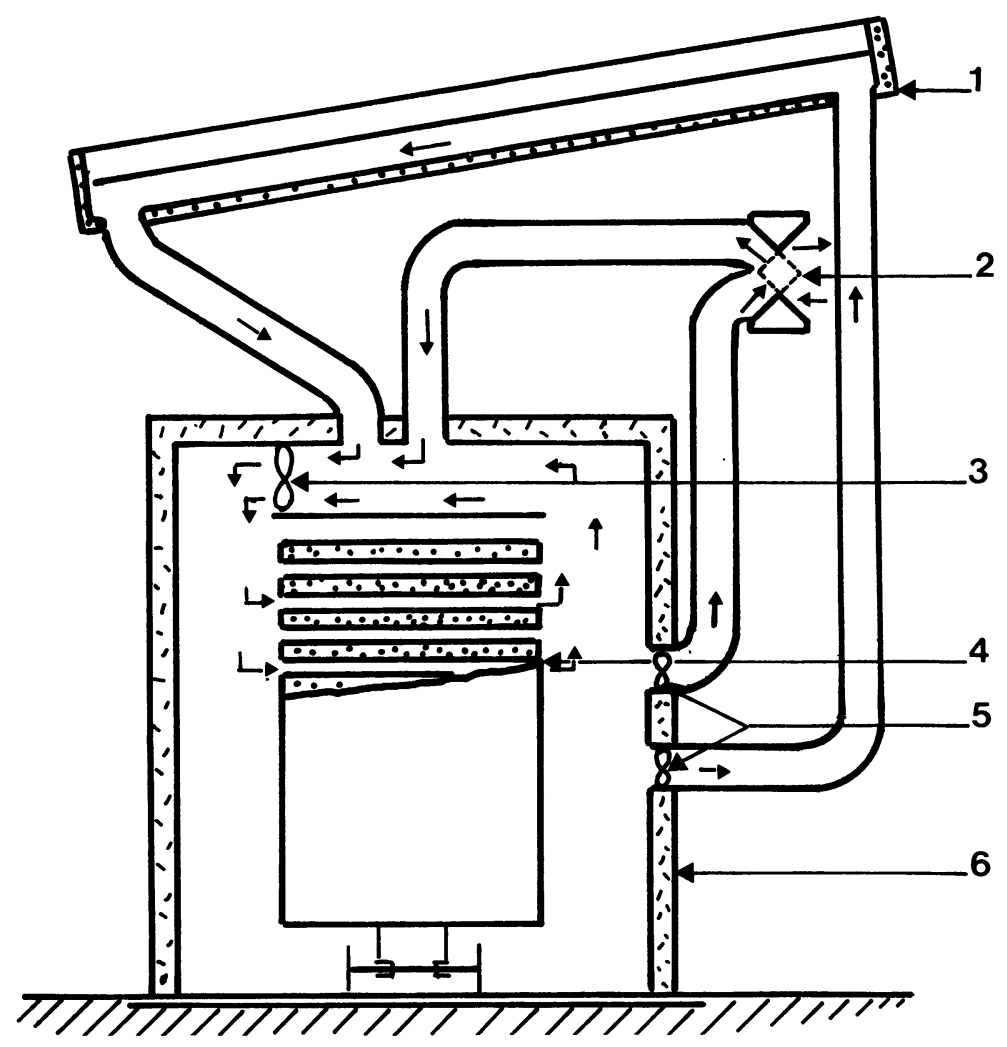

Fig. 1. - Schéma de principe du séchoir solaire installé durant l'été 1985 dans la zone portuaire de Kourou (Guyane) ; surface des capteurs solaires : $75 \mathrm{~m}^{2}$, volume de la cellule : $65 \mathrm{~m}^{3}$, volume de bois séché : $10 \mathrm{~m}^{3}$.

[Schema of the solar timber dryer built in Kourou (French Guyana) during summer 1985. Insolator area : $75 \mathrm{~m}^{2}$, cell volume : $65 \mathrm{~m}^{3}$, wood volume : $10 \mathrm{~m}^{3}$.]

1. Capteurs solaires à air. 2. Echangeur thermique. 3. Ventilation cellule. 4. Pile de bois. 5. Ventilation de gaines.

6. Cellule isolée. 
sur une charpente spécifique en bois local. Là encore, ce sont des éléments industriels : absorbeur en tôle profilée, isolation par mousse de polyuréthane $(12 \mathrm{~cm})$, couverture en polyester protégé par du Tedlar assurant l'effet de serre.

\section{Hypothèse de modélisation.}

Nous allons donc traiter le problème en partant de l'équation générale de la vitesse de séchage.

Les variables impliquées - telles que la température, la teneur en eau de l'air et du bois, la vitesse de l'air et l'épaisseur du bois $E_{\mathrm{b}}$, la teneur en eau initiale du bois et la teneur en eau finale ou à l'équilibre -, sont considérées comme des fonctions continues dans l'espace et le temps pour le domaine d'application envisagé. Nous retiendrons les moyennes volumiques des distributions des variables microscopiques correspondantes. Le choix de la température de l'air n'intervient qu'indirectement dans le phénomène de migration de l'eau: elle conditionne la température du bois qui influe sur le transfert des molécules dans le matériau.

Nous les prenons en compte dans l'équation de type :

$$
\frac{\mathrm{d} N_{\mathrm{b}}}{\mathrm{d} t}=F\left(T_{\mathrm{a}}, N_{\mathrm{a}}, V_{\mathrm{a}}, T_{\mathrm{b}}, N_{\mathrm{b}}, N_{\mathrm{eq}}, E_{\mathrm{b}}, N_{0}\right) .
$$

De plus, nous ne prendrons pas en compte une éventuelle réhumidification du produit, même s'il y a condensation sur celui-ci. Dans ce dernier cas, la vitesse de séchage sera considérée comme nulle.

Notre démarche repose alors sur deux hypothèses :

1) Le flux massique de l'eau du bois dans l'air a lieu sous l'action d'un terme moteur choisi comme étant la différence entre la teneur en eau du matériau et celle qu'il aurait à l'équilibre hygroscopique avec son environnement.

2) Seuls les paramètres de séchage qui subiront des variations significatives dans l'application de ce modèle ont été pris en compte. Nous considèrerons que chacun d'eux a une influence indépendante des autres.

Nous allons maintenant expliciter ces deux concepts.

2.1 TERME MOTEUR DE SÉCHAGE. - La vitesse de séchage est souvent exprimée comme étant fonction d'un terme choisi comme moteur de la migration de l'eau. Physiquement ce terme peut être un gradient de pression de vapeur et/ou de teneur en eau du matériau.

Nous avons retenu ici la valeur la plus facilement mesurable : le poids de l'échantillon qui nous donne sa teneur en eau moyenne. La valeur intervenant comme limite est la teneur en eau à l'équilibre avec l'air $\left(N_{\mathrm{eq}}\right)$.
La relation proposée est donc :

$\frac{\mathrm{d} N_{\mathrm{b}}}{\mathrm{d} t}=F\left(T_{\mathrm{a}}, H_{\mathrm{a}}, V_{\mathrm{a}}, T_{\mathrm{b}}, N_{\mathrm{b}}, E_{\mathrm{b}}, N_{0}\right)\left(N_{\mathrm{b}}-N_{\mathrm{eq}}\right)$.

La forme de cette relation (2.2) a déjà été trouvée par Danze et Bougnet pour des planches de peuplier de $25 \mathrm{~mm}$ (Villière [2]).

Une telle relation permet d'annuler le flux massique lorsque le bois est à l'équilibre avec son environnement (terme moteur nul).

\subsection{INFLUENCE DES PARAMÈTRES DE SÉCHAGE. -} Nous nous proposons ici de passer en revue l'influence des principaux paramètres de séchage sur la durée et la vitesse de séchage du bois.

Le peu de données sur les formes analytiques des fonctions invariantes donnant le flux massique nous amène à faire une analogie entre celles-ci et le coefficient de transport par diffusivité $\left(K_{N}\right)$ couplé au gradient de teneur en eau $\frac{\partial N_{\mathrm{b}}}{\partial X}$;

$K_{N}$ est lui-même lié au coefficient de diffusion massique $D_{\mathrm{m}}$, par la relation :

$$
K_{N}=D_{\mathrm{m}} \frac{\partial C}{\partial N_{\mathrm{b}}} .
$$

Pour un profil de teneur en eau parabolique dans une plaque d'épaisseur $E_{\mathrm{b}}$, la teneur en eau à la surface est donnée par Berger et Pei [3].

$$
N_{\mathrm{b} \text { surf }}=N_{\mathrm{b}}+E_{\mathrm{b}} \cdot \frac{2}{3}\left(\frac{\partial N_{\mathrm{b}}}{\partial X}\right)_{\text {surf }}
$$

avec

$$
\frac{\mathrm{d} N_{\mathrm{b}}}{\mathrm{d} t}=-K_{N} \frac{\partial N_{\mathrm{b}}}{\partial x}
$$

d'où

$$
N_{\text {b. surf }}=N_{\mathrm{b}}-\frac{K_{1}}{K_{N}} \frac{\mathrm{d} N_{\mathrm{b}}}{\mathrm{d} t} \quad\left(K_{1}: \text { constante }\right)
$$

et

$$
\frac{\mathrm{d} N_{\mathrm{b}}}{\mathrm{d} t}=\frac{K_{N}}{K_{1}}\left(N_{\mathrm{b}}-N_{\mathrm{b} . \text { surf }}\right)
$$

si l'on suppose la surface du produit proche de l'équilibre, on a :

$$
\frac{\mathrm{d} N_{\mathrm{b}}}{\mathrm{d} t}=\frac{K_{N}}{K_{1}}\left(N_{\mathrm{b}}-N_{\mathrm{eq}}\right) .
$$

Etant donné le précédent choix du terme moteur, nous montrons ainsi l'analogie entre la fraction $\left(K_{N} / K_{1}\right)$ variant comme un coefficient de diffusion massique, et la fonction des paramètres du séchage que nous allons définir comme le produit de fonctions à variables séparées. 


$$
F\left(X_{i}\right)=\prod_{i=1}^{n} F\left(X_{i}\right)
$$

avec

$X_{i}$ : paramètre de séchage

$n$ : nombre de paramètres considérés.

2.2.1 Température de l'air. - Ce paramètre a une influence primordiale sur la température à l'intérieur du produit et sur la cinétique de séchage. Plus la température de l'air est élevée, plus la température du bois l'est, et plus les cinétiques sont rapides. Dans les essais sur échantillons de faible inertie on peut confondre la température du bois et la température de l'air.

Il est courant de considérer que le flux massique suit la même loi de variation en fonction de la température que le coefficient de diffusivité massique ; c'est-à-dire une forme d'Arrhenius :

$$
D_{\mathrm{m}}=D_{0} \exp \left(-E / R_{\mathrm{v}} T_{\mathrm{k}}\right)
$$

avec $E$ : Energie d'activation.

Nous utiliserons une forme semblable pour la fonction invariante de la température.

$$
F\left(T_{\mathrm{a}}\right)=\exp \left(f+g / T_{\mathrm{a}}\right)
$$

$f$ et $g$ : paramètres à identifier $(g<0)$.

2.2.2 Teneur en eau du bois. - Avec la température, c'est le paramètre le plus influent sur la vitesse de séchage. Les courbes représentant le coefficient de diffusion en fonction de ce paramètre ne sont pas nombreuses.

En 1982, Moyne [4] signale que le coefficient de diffusion massique peut être considéré comme une fonction du second degré de la teneur en eau.

Notre choix se porte sur une fonction du second degré.

$$
F\left(N_{\mathrm{b}}\right)=a+b \cdot N_{\mathrm{b}}+c \cdot N_{\mathrm{b}}^{2}
$$

$a, b, c,:$ paramètres à identifier.

2.2.3 Dimension des planches. - En début de séchage, la vitesse est inversement proportionelle à l'épaisseur, et en fin de séchage au carré de l'épaisseur (Villière [2]).

Kollmann [5] explique que la vitesse de diffusion est proportionnelle au gradient de pression de vapeur, et que ce gradient est inversement proportionnel à l'épaisseur des planches. La vitesse de séchage est donc inversement proportionnelle à l'épaisseur et le temps de séchage proportionnel au carré de l'épaisseur.

Dans notre cas, nous approximons par une fonction linéaire l'influence de l'épaisseur :

$$
F\left(E_{\mathrm{b}}\right)=d+e \cdot E_{\mathrm{b}}
$$

$d$ et $e$ : paramètres à identifier $(e<0)$.
2.2.4 Autres paramètres. - La vitesse de l'air n'a un rôle significatif qu'en début de séchage. En fin, sa valeur influence peu la cinétique. En fait elle aura une valeur fixe dans le séchoir en moyenne $2 \mathrm{~m} / \mathrm{s}$ au voisinage des pièces de bois ; en gardant cette même valeur dans les expérimentations nous n'avons pas à étudier son influence ;

- l'humidité de l'air. Son rôle est faible sauf peut être en début de séchage. Elle sera prise en compte dans le calcul de la teneur en eau à l'équilibre.

- La répartition interne de la teneur en eau du bois en cours de séchage, a une influence aussi importante sur le séchage que son intégrale à n'importe quel instant.

Batsale [6] dans son étude sur le séchage des noisettes en tient compte. En fait dans ce travail nous nous limiterons à des mesures de teneur en eau moyenne c'est-à-dire à une approche plus globale.

Par contre la répartition initiale doit être uniforme. Elle est d'autre part pratiquement constante pour les essences considérées $(60 \%$ base matière sèche).

En conclusion l'équation finalement choisie est de la forme :

$$
\frac{\mathrm{d} N_{\mathrm{b}}}{\mathrm{d} t}=F_{1}\left(N_{\mathrm{b}}\right) \cdot F_{2}\left(E_{\mathrm{b}}\right) \cdot F_{3}\left(T_{\mathrm{a}}\right) \cdot\left(N_{\mathrm{b}}-N_{\mathrm{eq}}\right) .
$$

2.3 IDENTIFICATION DES COEFFICIENTS POUR UNE ESSENCE DE RÉFÉRENCE : L'ANGÉLIQUE (DICORYNA GUIANENSIS). - Les coefficients du modèle évaporatoire sont dans ce chapitre identifiés pour l'essence ayant servi aux essais. Nous adapterons ce modèle à d'autres essences par la suite. Les échantillons étant recouverts de film d'aluminium sur leurs faces latérales, nous avons négligé l'évaporation par ces faces.

Le tunnel de séchage de laboratoire [7] ayant servi aux essais comporte :

- un couloir d'humidification et de chauffage de l'air contrôlé,

- un ventilateur de mise en vitesse,

- une cellule de séchage proprement dite.

Ses plages d'utilisation sont :

— vitesse de l'air : de 0 à $2,5 \mathrm{~m} \mathrm{~s}^{-1}$,

- température de l'air: de la température ambiante à $70^{\circ} \mathrm{C}$,

- teneur en eau de l'air : de la valeur ambiante à $30 \mathrm{~g} \mathrm{eau} / \mathrm{kg}$ air sec.

La variation d'humidité du produit, outre la pesée classique, peut être mesurée en continu grâce à la déflexion statique de l'échantillon relié à un pont de jauges (précision 0,5\% charge limite $300 \mathrm{~g}$ ).

Détermination de la vitesse de séchage.

Sur la courbe de perte de masse au cours du temps, nous effectuons les lissages successifs des points expérimentaux, par groupe de cinq en utilisant 
Tableau I. - Identification des coefficients pour l'angélique.

\begin{tabular}{|c|c|}
\hline Valeur de la fonction & Domaine d'utilisation \\
\hline$F\left(N_{\mathrm{b}}\right)=\left[5,24-0,22 N_{\mathrm{b}}-0,0031 N_{\mathrm{b}}^{2}\right] / 3600$ & $\begin{array}{c}N_{\mathrm{b}} \text { en } \% \\
20<N_{\mathrm{b}}<60 \%\end{array}$ \\
\hline$F\left(E_{\mathrm{b}}\right)=3-E_{\mathrm{b}} / 0,012$ & $\begin{array}{c}E_{\mathrm{b}} \text { en m } \\
0,012<E_{\mathrm{b}}<0,032\end{array}$ \\
\hline$F\left(T_{\mathrm{a}}\right)=\exp \left(0,84-47,45 / T_{\mathrm{a}}\right)$ & $\begin{array}{c}T_{\mathrm{a}} \text { en }{ }^{\circ} \mathrm{C} \\
20<T_{\mathrm{a}}<65\end{array}$ \\
\hline
\end{tabular}

une régression polynomiale du second degré (méthode des moindres carrés). A chaque fois, la valeur de la dérivée au point central nous donne la vitesse de séchage.

\section{Relation finale.}

Nous avons ainsi identifié dans un domaine d'utilisation donné, les sept coefficients entrant dans les trois fonctions invariantes figurant dans l'expression de la vitesse de séchage de l'Angélique. Les résultats numériques sont rassemblés dans le tableau I.

2.4 VÉRIFICATION DU MODÈLE. - Les résultats obtenus par le modèle précédent, de la vitesse de séchage ont été validés par des mesures, effectuées en étuve à atmosphère contrôlée sur des échantillons d'Angélique, lors d'expériences n'ayant pas servi à l'identification. Les conditions de séchage sont constantes pendant la durée de l'opération.

L'allure des courbes obtenues (Fig. 2), vitesse de séchage et teneur en eau du bois en fonction du temps, est satisfaisante.

- la courbe théorique se situe au centre de la bande de dispersion des points expérimentaux;

- la présence d'une phase à vitesse de séchage constante n'est pas mise en évidence en accord avec nos hypothèses ;

- la teneur en eau du bois tend bien asymptotiquement vers sa teneur d'équilibre ;

- l'erreur enregistrée est inférieure à $8 \%$ ce qui correspond à nos objectifs pour une première confrontation d'une partie du modèle du séchoir avec la réalité du séchage.

Nous pensons par la suite comparer la simulation de la cinétique avec d'autres mesures, en particulier en utilisant des quantités de bois plus importantes.

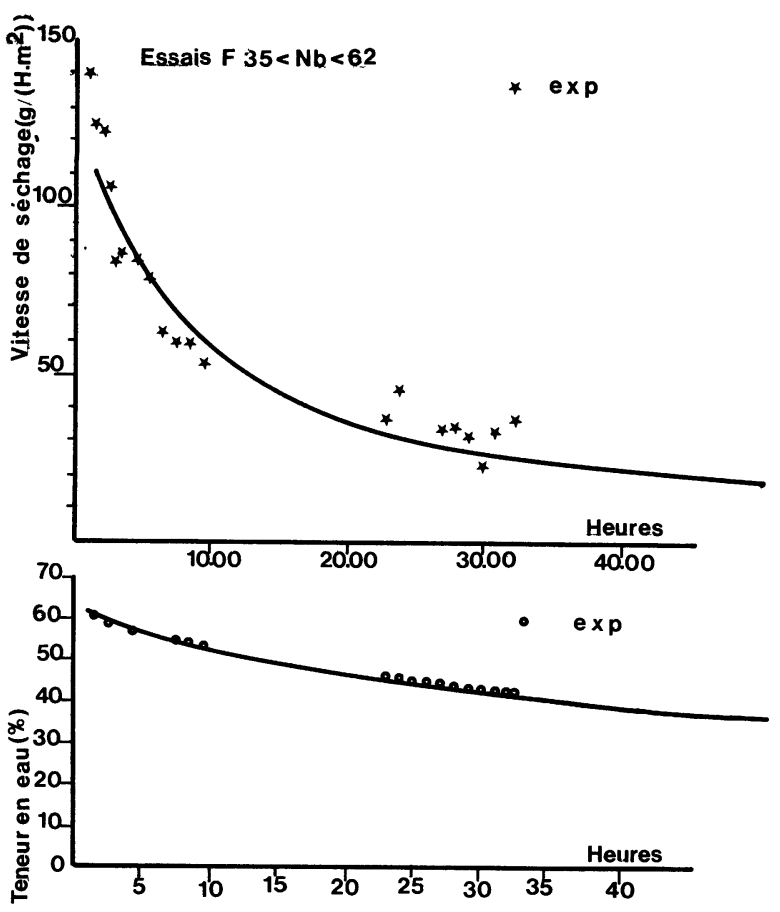

Fig. 2. - Comparaison des résultats théoriques et expérimentaux.

[Comparison of experimental and theoretical values.]

Dans ce cas l'hypothèse sur la température de l'échantillon n'est plus vraie: l'inertie thermique d'une pile de bois n'est en effet pas négligeable. Nous devons prendre en compte la température du bois dans le modèle évaporatoire et non plus celle de l'air. La fonction $F\left(T_{\mathrm{b}}\right)$ s'écrit alors

$$
F\left(T_{\mathrm{b}}\right)=\exp \left(0,84-47,45 / T_{\mathrm{b}}\right) .
$$

Cette température $T_{\mathrm{b}}$ est une variable calculée par une équation de bilan énergétique de la forme.

$\left[\begin{array}{c}\text { variation d'énergie } \\ \text { interne du bois }\end{array}\right]+\left[\begin{array}{c}\text { énergie de vaporisation } \\ \text { de l'eau du bois }\end{array}\right]=\left[\begin{array}{c}\text { échange convectif } \\ \text { air-bois }\end{array}\right]$. 
Nous sommes donc amenés à résoudre le système suivant :

$$
\left\{\begin{aligned}
M_{\mathrm{c}} c p_{\mathrm{b}} \frac{\mathrm{d} T_{\mathrm{b}}}{\mathrm{d} t} & =-L_{\mathrm{v}} \frac{\mathrm{d} M_{\mathrm{b}}}{\mathrm{d} t}+\alpha_{\mathrm{a}-\mathrm{b}} \cdot S_{\mathrm{b}} \cdot\left(T_{\mathrm{a}}-T_{\mathrm{b}}\right) \\
\frac{\mathrm{d} N_{\mathrm{b}}}{\mathrm{d} t} & =\left(a+b \cdot N_{\mathrm{b}}+c \cdot N_{\mathrm{b}}^{2}\right)\left(d+e \cdot E_{\mathrm{b}}\right) \cdot \exp \left(f+g / T_{\mathrm{b}}\right)\left(N_{\mathrm{b}}-N_{\mathrm{eq}}\right) .
\end{aligned}\right.
$$

La prochaine étape du travail expérimental est donc la validation du modèle sur un chargement réel de bois, dans des conditions climatiques réelles donc aléatoires puisque l'énergie qui doit être utilisée est l'énergie solaire - Différentes essences de bois doivent également être traitées [8].

\section{Modélisation du séchoir.}

Seuls, les phénomènes énergétiques sont pris en compte : transfert de chaleur et de masse. L'étude aéraulique sort du cadre de ce travail, bien que les vitesses et pertes de charges aient été évaluées pour la conception.

Nous avons dans un premier temps envisagé le séchoir dans sa version la plus simple, en le décomposant en deux parties étudiées indépendamment : la cellule et les capteurs solaires. Le vecteur énergétique assurant la liaison entre les différents éléments solides est le volume d'air. La modélisation des capteurs solaires a été détaillée par Pallet en 1985 [8].

Nous présentons ici uniquement la modélisation du caisson de dessication.

\subsection{LES ÉQUATIONS.}

3.2.1 Le bois. - La vitesse de variation de la température du bois s'écrit en fonction des échanges avec son environnement :

$$
\begin{gathered}
M_{\mathrm{b}} \cdot C p_{\mathrm{b}} \cdot \frac{\mathrm{d} T_{\mathrm{b}}}{\mathrm{d} t}=-L v_{\mathrm{b}-\mathrm{a}} \cdot \frac{\mathrm{d} M_{\mathrm{b}-\mathrm{a}}}{\mathrm{d} t}+\alpha \alpha_{\mathrm{a}-\mathrm{b}} \cdot S_{\mathrm{b}} \cdot\left(T_{\mathrm{a}}-T_{\mathrm{b}}\right) \\
{\left[\begin{array}{c}
\text { Variation d'énergie } \\
\text { interne du bois }
\end{array}\right]=\left[\begin{array}{c}
\text { Energie de migration } \\
\text { évaporation de l'eau } \\
\text { du bois }
\end{array}\right]+\left[\begin{array}{c}
\text { Échanges par convection } \\
\text { entre l'air et le bois }
\end{array}\right]}
\end{gathered}
$$

$\frac{\ll \mathrm{d} M_{\mathrm{b}-\mathrm{a}} »}{\mathrm{~d} t}$ est la vitesse d'évaporation de l'eau du bois, c'est une des caractéristique du matériau dans son environnement.

La conservation de la masse d'eau contenue dans le bois s'écrit :

$$
N_{\mathrm{b}(t)}=N_{\mathrm{b}(t-\delta t)}-\left(\frac{\mathrm{d} M_{\mathrm{b}-\mathrm{a}}}{\mathrm{d} t} / M_{\mathrm{s}}\right)_{(t-\delta t)} \cdot \delta t .
$$

3.2.2 La cellule. - L'inertie thermique de la cellule étant non négligeable devant celle de la pile de bois, l'équation différentielle décrivant ses variations de température est :

$$
M_{\mathrm{p}} \cdot C p_{\mathrm{p}} \cdot \frac{\mathrm{d} T_{\mathrm{p}}}{\mathrm{d} t}=S_{\mathrm{p}} \cdot \alpha_{\mathrm{a}-\mathrm{p}} \cdot\left(T_{\mathrm{a}}-T_{\mathrm{p}}\right)-S_{\mathrm{ex}} \cdot \lambda_{\mathrm{ep}} \cdot\left(T_{\mathrm{ex}}-T_{\mathrm{p}}\right)+L c_{\mathrm{a}-\mathrm{p}} \frac{\mathrm{d} M_{\mathrm{a}-\mathrm{p}}}{\mathrm{d} t}
$$




$$
\left[\begin{array}{c}
\text { Variation d'énergie } \\
\text { interne de la } \\
\text { cellule }
\end{array}\right]=\left[\begin{array}{c}
\text { Echange convectif } \\
\text { entre l'air et } \\
\text { les parois }
\end{array}\right]-\left[\begin{array}{c}
\text { Pertes } \\
\text { conductives } \\
\text { par les parois }
\end{array}\right]+\left[\begin{array}{c}
\text { Condensation } \\
\text { de l'eau de l'air } \\
\text { sur les parois }
\end{array}\right]
$$

$\lambda \quad$ représente la conductivité des parois d'épaisseur ep.

$L c_{\mathrm{a}-\mathrm{p}}$ représente l'énergie de condensation de l'eau sur les parois.

$\frac{\mathrm{d} M_{\mathrm{a}-\mathrm{p}}}{\mathrm{d} t}$ représente la vitesse de condensation de l'eau sur les parois.

3.2.3 L'air. - L'inertie thermique de l'air est insignifiante devant celle du bois. La constante de temps de l'équation décrivant le bilan énergétique est donc, pour l'air, infiniment plus petite que celle du bois.

D'autre part la cellule est un système ouvert puisque reliée à l'extérieur grâce au ventilateur de renouvellement d'air et aux capteurs solaires ventilés par leur propre ventilateur. On peut écrire :

+ Le bilan d'enthalpie des variations d'enthalpie pendant un intervalle de temps $\delta t$

$$
\begin{aligned}
& {\left[\begin{array}{c}
\text { variation d'enthalpie } \\
\text { de l'air entrant et } \\
\text { sortant }
\end{array}\right]=\left[\begin{array}{c}
\text { variation d'enthalpie } \\
\text { de l'air intérieur }
\end{array}\right]+\left[\begin{array}{c}
\text { pertes convectives } \\
\text { (vers parois et } \\
\text { vers bois }
\end{array}\right]-\left[\begin{array}{c}
\text { énergie de condensation } \\
\text { eau de l'air/parois }
\end{array}\right] } \\
& H_{\mathrm{a}}\left(M_{\mathrm{a}}, N_{\mathrm{a}}, T_{\mathrm{a}}\right)_{t}= H_{\mathrm{a}}\left(M_{\mathrm{a}}, N_{\mathrm{a}}, T_{\mathrm{a}}\right)_{t-\delta t}-\left(S_{\mathrm{b}}, \alpha_{\mathrm{a}-\mathrm{b}}\left(T_{\mathrm{a}}-T_{\mathrm{b}}\right)+S_{\mathrm{p}} \alpha_{\mathrm{a}-\mathrm{p}}\left(T_{\mathrm{a}}-T_{\mathrm{p}}\right)\right) \cdot \delta t \\
&-L c_{\mathrm{a}-\mathrm{p}}\left(T_{\mathrm{p}}\right) \cdot \mathrm{d} M_{\mathrm{a}-\mathrm{p}} \\
&+H_{\mathrm{ee}}\left(M_{\mathrm{r}}, N_{\mathrm{ex}}, T_{\mathrm{ex}}\right)+H_{\mathrm{ec}}\left(M_{\mathrm{c}}, N_{\mathrm{a}}, T_{\mathrm{sc}}\right) \\
&-H_{\mathrm{se}}\left(M_{\mathrm{r}}, N_{\mathrm{a}}, T_{\mathrm{a}}\right)-H_{\mathrm{sc}}\left(M_{\mathrm{c}}, N_{\mathrm{a}}, T_{\mathrm{a}}\right)
\end{aligned}
$$

+ Le bilan des masses d'eau contenues dans l'air échangées pendant l'intervalle de temps $\delta t$

$\left[\begin{array}{c}\text { variation teneur } \\ \text { en eau de l'air } \\ \text { de la cellule }\end{array}\right]=\left[\begin{array}{c}\text { évaporation } \\ \text { eau du bois }\end{array}\right]-\left[\begin{array}{c}\text { condensation sur } \\ \text { parois cellules }\end{array}\right]+\left[\begin{array}{c}\text { eau de l'air extérieur } \\ \text { entrant }\end{array}\right]-\left[\begin{array}{c}\text { eau de l'air } \\ \text { sortant }\end{array}\right]$

$$
\left(M_{\mathrm{a}} \cdot N_{\mathrm{a}}\right)_{t}=\left(M_{\mathrm{a}} \cdot N_{\mathrm{a}}\right)_{t-\delta t}+\left(\mathrm{d} M_{\mathrm{a}-\mathrm{b}} / \mathrm{d} t-\mathrm{d} M_{\mathrm{a}-\mathrm{p}} / \mathrm{d} t\right)_{t-\delta t} \cdot \delta t+M_{\mathrm{r}} \cdot\left(N_{\mathrm{ex}}-N_{\mathrm{a}}\right)_{t-\delta t} \cdot \delta t .
$$

La discrétisation du système des cinq équations différentielles précédentes nous donne des bilans énergétiques sur des pas de temps petit $(\delta t)$. La valeur prise pour « $\delta t »$ est égale au temps nécessaire au volume d'air contenu dans un tiers du séchoir pour passer dans un ventilateur de brassage.

Ces équations sont découplées et résolues par une méthode d'Euler explicite. En partant des conditions à l'instant $\left\langle t_{0} »\right.$, on calcule les masses et les énergies échangées, puis les valeurs des cinq inconnues:

$$
T_{\mathrm{b}(t)}, N_{\mathrm{b}(t)}, T_{\mathrm{p}(t)}, T_{\mathrm{a}(t)} \text { et } N_{\mathrm{a}(t)} \text { à l'instant } t_{0}+\delta t .
$$

De nombreux termes, propres aux transferts thermiques et massiques interviennent, nous allons les expliciter dans le cas particulier de cette cellule.

\subsection{Paramètres propres À la cellule.}

3.3.1 Transferts thermiques. - La puissance transmise par convection s'écrit sous la forme générale :

$$
P_{\text {conv }}=\alpha \cdot S . \delta T .
$$

Le coefficient de convection « $\alpha$ » est calculé à partir du nombre de Nusselt :

$$
N u=\alpha \cdot D_{\mathrm{h}} / \lambda
$$

$D_{\mathrm{h}}$ est une longueur caractéristique.

* Echange air-bois : $\alpha_{\mathrm{a}-\mathrm{b}}$ et $S_{\mathrm{B}}$.

Les coefficients d'échange de chaleur et de matière entre le bois et l'air et entre les parois et l'air dépendent étroitement du type d'écoulement réalisé. Il est évident que dans une telle pile, les vitesses et donc les nombres de Reynolds ne sont pas partout les mêmes. Une connaissance détaillée des fluctuations de l'écoulement dans le cas de cette géométrie, bien qu'importante, sort du cadre de cette étude. Nous nous contenterons des valeurs moyennes à partir desquelles nous déterminerons les coefficients d'échanges.

A ce niveau, l'air circule entre deux plaques parallèles; le nombre de Reynolds donne une idée sur le régime de l'écoulement :

$$
R e=\frac{V_{\mathrm{a}} \cdot D_{\mathrm{h}}}{\nu}
$$

$D_{\mathrm{h}}=$ diamètre hydraulique

$=2 x$ épaisseur de l'écoulement ou $2 x$ intervalle entre planches

$\nu=$ viscosité cinématique de l'air $\left(\right.$ à $42^{\circ} \mathrm{C}$, $\left.\nu=1,72 \times 10^{-5} \mathrm{~m}^{2} / \mathrm{s}\right)$. 
Nous obtenons un nombre de Reynolds de l'ordre de $5800\left(E_{\mathrm{b}}=25 \mathrm{~mm}\right)$ à $7000\left(E_{\mathrm{b}}=30 \mathrm{~mm}\right)$. A ces valeurs, l'écoulement sera certainement turbulent entre les planches.

Pour calculer le nombre de Nusselt, nous avons repris la corrélation proposée par Dittus et Boelter [10]) pour ce type de géométrie.

$N u=0,023 \cdot R e^{0,8} \cdot \operatorname{Pr}^{0,4}$ avec $P r=0,7$ (nombre de Prandtl).

Tableau II. - Valeurs du coefficient de transfert de chaleur pour deux épaisseurs de planches.

\begin{tabular}{c|l|c}
$E_{\mathrm{b}}$ en mm & $N u$ & $\alpha_{\mathrm{a}-\mathrm{b}}\left(\mathrm{W} / \mathrm{m}^{2} . \mathrm{K}\right)$ \\
\hline 25 & 20,5 & 11,2 \\
30 & 24 & 10,9
\end{tabular}

* Echange air-parois : $\alpha_{\mathrm{a}-\mathrm{p}}$ et $S_{\mathrm{p}}$

L'inertie de tous les éléments constitutifs des parois est répartie sur leur surface interne. La température est définie à ce niveau. Les pertes se font à partir de cette face, à travers l'isolant.

Les résistances thermiques valent :

- face intérieure de la cellule : $R_{1}=1 / \alpha_{\mathrm{a}-\mathrm{p}}$

En prenant comme nombre de Nusselt [9] $N u=0,036 \cdot \operatorname{Pr}^{1 / 3} \cdot \operatorname{Re}^{4 / 5}$

On trouve pour les faces de la cellule :

- Parois verticales

$1=4 \mathrm{~m} \rightarrow N u=1095 \rightarrow \alpha_{\mathrm{a}-\mathrm{p}}=7,50 \mathrm{~W} / \mathrm{m}^{2} \cdot \mathrm{K}$

- Plafond

$1=3,5 \mathrm{~m} \rightarrow N u=984 \rightarrow \alpha_{\mathrm{a}-\mathrm{p}}=7,73 \mathrm{~W} / \mathrm{m}^{2} \cdot \mathrm{K}$

- traversée de la paroi : $R_{2}=\mathrm{ep} / \lambda_{\mathrm{p}}$

Le coefficient de conduction des parois est dans notre cas :

$$
\lambda_{\mathrm{p}}=0,022 \mathrm{~W} / \mathrm{m} \cdot \mathrm{K} \text { (polyuréthane). }
$$

La résistance de la face extérieure, $R_{3}$, est certainement du même ordre de grandeur que celle de la face intérieure $R_{1}$.

L'ensemble des parois oppose donc aux transferts thermiques entre l'intérieur et l'extérieur de la cellule une résistance :

$$
\sum R=R_{1}+R_{2}+R_{3}
$$

En définitive, la valeur du coefficient global des pertes est :

$$
\alpha_{\mathrm{a}-\mathrm{ex}}=0,18 \mathrm{~W} / \mathrm{m}^{2} \cdot \mathrm{K} .
$$

\subsubsection{Transferts massiques.}

* Echange bois-air : $L v_{\mathrm{b}-\mathrm{a}}$ et $\frac{\mathrm{d} M_{\mathrm{b}-\mathrm{a}}}{\mathrm{d} t}$.
L'énergie de vaporisation de l'eau est la somme de :

- l'énergie de vaporisation de l'eau libre : $L v$,

- la chaleur nécessaire pour vaincre les forces d'attraction entre le bois et l'eau : (Kollmann [5]).

La vitesse d'évaporation de l'eau du bois a été déterminée à partir de l'équation générale du séchage déjà établie.

$$
\begin{aligned}
& \frac{\mathrm{d} M_{\mathrm{b}-\mathrm{a}}}{\mathrm{d} t}=M_{\mathrm{s}} \cdot\left(a+b \cdot N_{\mathrm{b}}+c \cdot N_{\mathrm{b}}^{2}\right) \times \\
& \quad \times\left(d+e \cdot E_{\mathrm{b}}\right) \cdot \exp \left(f+g / T_{\mathrm{b}}\right) \cdot\left[N_{\mathrm{b}}-N_{\mathrm{eq}}\right] .
\end{aligned}
$$

* Echange air-parois : $L c_{\mathrm{a}-\mathrm{p}}$ et $\frac{\mathrm{d} M_{\mathrm{a}-\mathrm{p}}}{\mathrm{d} t}$.

La théorie de Nusselt est utilisée [9].

Lors de la condensation d'une quantité d'eau $\ll \mathrm{d} m$ » sur la paroi, la chaleur transmise à la paroi est :

$$
\mathrm{d} q=L c_{\mathrm{a}-\mathrm{p}} \cdot \mathrm{d} m
$$

avec: $L c_{\mathrm{a}-\mathrm{p}}=L v+0,68 \cdot \mathrm{Cp}\left(T_{\text {sat }}-T_{\mathrm{p}}\right)$.

Etant donné les niveaux de température considérés, la correction due au sous-refroidissement du liquide peut être négligée.

La vitesse de condensation de l'eau sur les parois peut se calculer par la méthode suivante :

- épaisseur du film d'eau du bas des parois

$$
E_{\mathrm{fe}}=\left[\frac{4 \nu(T) \cdot \lambda_{1}(T) \cdot H H \cdot\left(T_{\mathrm{sat}}-T_{\mathrm{p}}\right)}{g \cdot\left(\rho_{1}-\rho_{\mathrm{v}}\right) \cdot L c_{\mathrm{a}-\mathrm{p}}}\right]^{1 / 4}
$$

avec $H H$ : hauteur des parois de la cellule.

- débit massique au bas des parois par unité de largeur

$$
\dot{m}_{\mathrm{H}}=\frac{g \cdot\left[\rho_{1} \cdot\left(T_{\mathrm{m}}\right)-\rho_{\mathrm{v}} \cdot\left(N_{\mathrm{a}}, T_{\mathrm{a}}\right)\right]}{3 \cdot \nu\left(T_{\mathrm{m}}\right)} \cdot E_{\mathrm{fe}}^{3}
$$

- vitesse totale de condensation

$$
\frac{\mathrm{d} M_{\mathrm{a}-\mathrm{p}}}{\mathrm{d} t}=\dot{m}_{\mathrm{H}} \cdot L L
$$

$L L$ : largeur des parois.

\section{Fonctionnement simulé du séchoir.}

Notre objectif ici n'étant pas d'optimiser un type de régulation, nous en proposons un, pour pouvoir simuler le séchoir en fonctionnement.

L'étude de tous les éléments du système et leur rassemblement dans un modèle de simulation ne sont pas suffisants pour simuler le fonctionnement : il faut en plus choisir un type de régulation.

Ce choix peut être très différent suivant que l'on considère seulement le rendement thermique de l'installation ou la conduite du séchage qui conditionne la qualité du bois. 


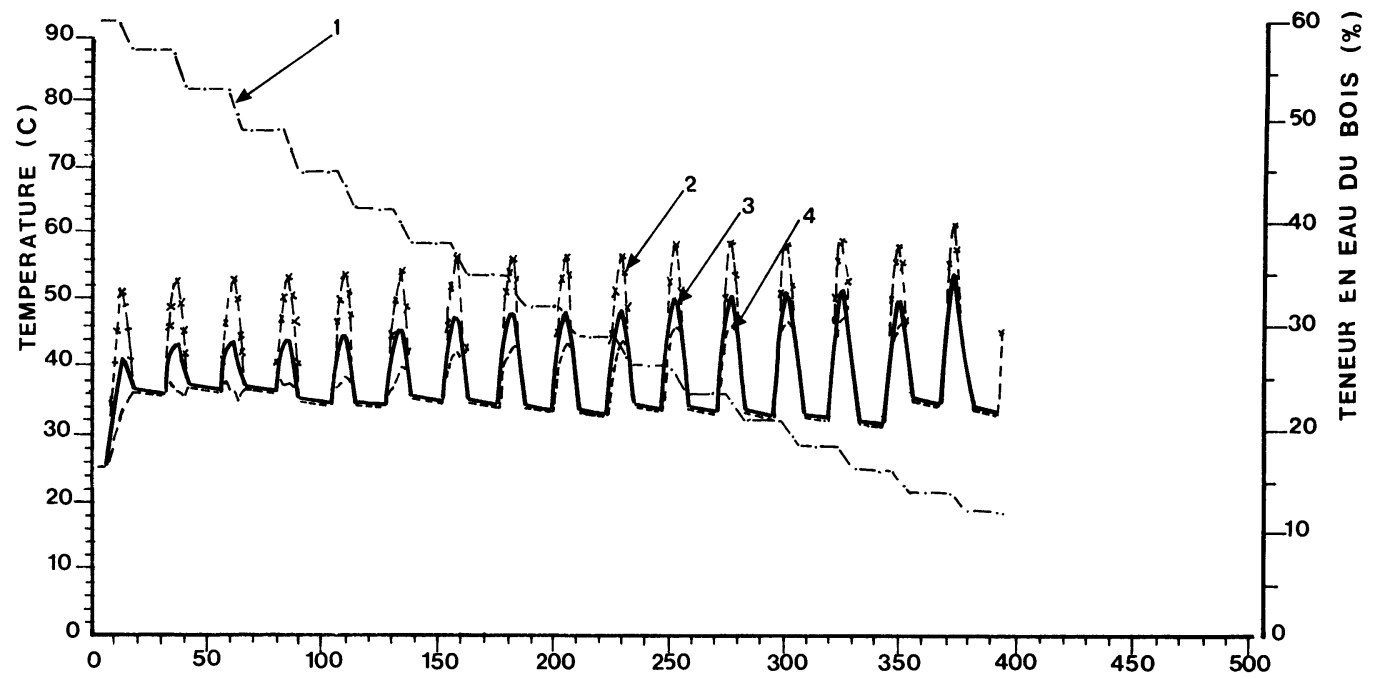

Fig. 3. - Simulation d'une opération de séchage solaire. Le bois séché est de l'angélique.

[A simulation of a solar drying cycle. Dryed wood is angelica.]

Dans un premier temps nous allons tester des régulations simples qui rentrent dans le programme sous forme d'algorithmes. Le rôle des variables qui interviennent dans ces régulations peut alors être mis en évidence.

On distingue :

- les variables primaires mesurées : température et humidité de l'air, masse et teneur en eau du bois, et l'ensoleillement,

- les variables secondaires calculées à partir des précédentes : pouvoir évaporatoire de ı'air, température du bois, gradient de séchage, rendements.
1. Teneur en eau du bois.

2. Température de l'air en sortie des capteurs.

3. Température de l'air dans la cellule.

4. Température du bois.

Sur l'axe horizontal le temps est exprimé en heures.

Plusieurs algorithmes de commande ont été implantés successivement dans notre modèle [8]. La figure 3 représente un exemple de simulation d'une opération de séchage au cours de laquelle la teneur en eau à l'équilibre $N_{\text {eq.cel }}$ de l'atmosphère de la cellule est comparée aux valeurs déduites de la table de séchage de l'Angélique, $N_{\text {eq.tab }}$. Lorsqu'il y a dépassement de cette valeur limite la mise en route du renouvellement d'air, permet de se re-situer dans des conditions convenables. Le rendement thermique moyen du séchoir est pendant les trois premiers jours de l'opération environ $40 \%$ (Pallet [8]).

On peut schématiser cet algorithme :

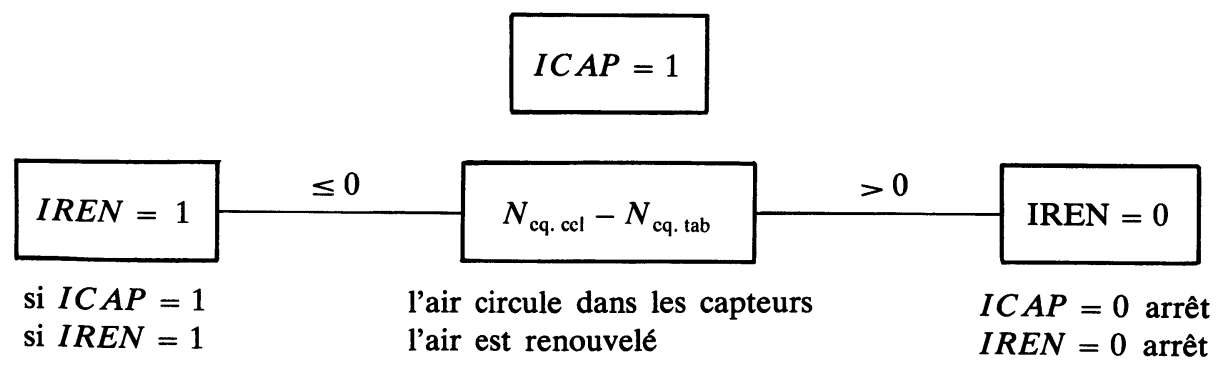

L'utilisation d'une rampe d'humidification de l'air doit permettre une plus grande souplesse dans la régulation du système.

Les courbes de simulation obtenues (Fig. 3) semblent correctes : le bois passe de $60 \%$ à $12 \%$ de teneur en eau en 16 jours.

Par contre le choix de l'algorithme de commande de la régulation peut avoir une grande importance sur la qualité du bois et sur le rendement thermique du séchoir.

\section{Conclusion. Objectifs et utilisation du modèle.}

Les hypothèses simplificatrices adoptées nous ont conduit à une modélisation globale de chaque soussystème du séchoir (caisson, capteur). La liaison entre ces différents éléments par l'intermédiaire d'un vecteur énergétique l'air, conduit à un système dont la simulation informatique est parfaitement cohérente.

Dans tous les cas, pour être totalement crédibles 
nos résultats doivent être comparés à la réalité et éventuellement recalés. Le séchoir solaire réalisé à Kourou doit permettre cette validation.

D'autre part l'étude de tous les éléments du système et leur rassemblement suivant une architecture donnée ne sont pas suffisants pour simuler un fonctionnement réel : il faut en plus choisir un type de régulation tel que la qualité du bois et le rendement thermique de l'ensemble soient convenables.

Nous nous trouvons donc en présence :

- d'un besoin: un bois que l'on doit sécher suivant un processus propre à chaque essence, défini par une table de séchage établie par des professionnels du séchage du bois et à laquelle on peut faire entière confiance ;

- d'un outil expérimental: un séchoir solaire construit à Kourou suivant un cahier de charge imposé par les professionnels du bois ;

- d'un outil mathématique de simulation: il doit permettre une optimisation de la régulation :

- quelle est la conduite du séchoir la plus appropriée au produit à sécher dans les conditions expérimentales de Guyane?

- quels paramètres choisir pour les tests de régulation?

Ces deux outils doivent permettre de proposer une conduite de séchage adaptée à une demande précise des professionnels du bois : sécher différentes essences de bois dans un lieu donné.

Notre objectif est de répondre à cette demande : non seulement il justifie le travail présent mais il en constitue une suite logique.

\section{Bibliographie}

[1] Themelin, A., Conception d'un prototype de séchoir solaire pour bois d'auvre de Guyane. Etude CEEMAT-CTFT/CIRAD (1985).

[2] Villiere, A., Séchage des bois (Edition Dunod) (1953) 80-120.

[3] Berger, D., Pei, D. C. T., « Drying of hygroscopic capillary porous solids : a theoritical approach » J. Heat Mass Transfer 16 (1973) 293-302.

[4] MoYne, C., Contribution à l'étude du transfert simultané de chaleur et de masse au cours du séchage sous vide d'un bois résineux, Thèse $\mathrm{Di}$, INP de Lorraine (1982).

[5] Kollmann, F. F. P., Cote, W. A., Principles of wood science and technology (Springer VerlagNY Inc.) 1968.
[6] Batsale, J. C., Contribution à l'étude du séchage d'un amas de produit en coque, Thèse $3^{\mathrm{e}}$ cycle, Bordeaux I (1984).

[7] Puiggali, J. R., Dispositif de campagne pour le séchage solaire de produits agro-alimentaires, Thèse de $3^{\mathrm{e}}$ cycle, Bordeaux I (1981).

[8] Pallet, D., Modélisation et contrôle d'un séchoir solaire à bois, Thèse DI, Perpignan-Toulouse (1985).

[9] SACADURA, J. F., Initiation aux transferts thermiques (Editions Techniques et Documentation) 1982.

[10] Dittus, F. W., Boelter, L. M. K., Univ. Calif., Publ. Eng. 2 (1930) 443. 